\title{
Cavitation Inception on Microparticles: A Self-Propelled Particle Accelerator
}

\author{
Arora, M.; Ohl, C.-D.; Mørch, Knud Aage
}

Published in:

Physical Review Letters

Link to article, DOI:

10.1103/PhysRevLett.92.174501

Publication date:

2004

Document Version

Publisher's PDF, also known as Version of record

Link back to DTU Orbit

Citation (APA):

Arora, M., Ohl, C-D., \& Mørch, K. A. (2004). Cavitation Inception on Microparticles: A Self-Propelled Particle Accelerator. Physical Review Letters, 92(17), 174501. https://doi.org/10.1103/PhysRevLett.92.174501

\section{General rights}

Copyright and moral rights for the publications made accessible in the public portal are retained by the authors and/or other copyright owners and it is a condition of accessing publications that users recognise and abide by the legal requirements associated with these rights.

- Users may download and print one copy of any publication from the public portal for the purpose of private study or research.

- You may not further distribute the material or use it for any profit-making activity or commercial gain

- You may freely distribute the URL identifying the publication in the public portal

If you believe that this document breaches copyright please contact us providing details, and we will remove access to the work immediately and investigate your claim. 


\title{
Cavitation Inception on Microparticles: A Self-Propelled Particle Accelerator
}

\author{
Manish Arora, ${ }^{1}$ Claus-Dieter Ohl, ${ }^{1, *}$ and Knud Aage Mørch ${ }^{2}$ \\ ${ }^{1}$ Department of Applied Physics, Physics of Fluids, University of Twente, Postbus 217, 7500 AE Enschede, The Netherlands \\ ${ }^{2}$ Department of Physics and Center of Quantum Protein, Technical University of Denmark, DK-2800 Lyngby, Denmark
}

(Received 24 June 2003; published 29 April 2004)

\begin{abstract}
Corrugated, hydrophilic particles with diameters between 30 and $150 \mu \mathrm{m}$ are found to cause cavitation inception at their surfaces when they are exposed to a short, intensive tensile stress wave. The growing cavity accelerates the particle into translatory motion until the tensile stress decreases, and subsequently the particle separates from the cavity. The cavity growth and particle detachment are modeled by considering the momentum of the particle and the displaced liquid. The analysis suggests that all particles which cause cavitation are accelerated into translatory motion, and separate from the cavities they themselves nucleate. Thus, in the research of cavitation nuclei the link is established between developed cavitation bubbles and their origin.
\end{abstract}

DOI: 10.1103/PhysRevLett.92.174501

PACS numbers: $47.55 . \mathrm{Bx}$

Introduction.-When plain water is exposed to a sufficient tensile stress it ruptures, and explosively expanding bubbles develop. This phenomenon, commonly termed cavitation, is found to occur at very low tensile stress, while thermodynamic calculations predict the stress to be very high [1]. This discrepancy can be explained by weak spots-cavitation nuclei-being present in water. The nuclei might be free gas bubbles that are stabilized [2], but this idea is not satisfactorily supported experimentally. Alternatively they are interfacial voids at solid surfaces of particles or surrounding walls [3]. The existence of such nuclei has received substantial experimental and also theoretical support [4-10].

In this Letter, we focus on the dynamics of a cavity expanding rapidly from a spherical microparticle and we show that at tensile stress in bulk water large vapor cavities grow from small surface regions on solid particles. The high speed photographs shown depict the explosive growth and the - at first sight unexpectedprocess of detachment of the particle from the surface of the cavity. Thus the particle gains a speed of more than $10 \mathrm{~m} / \mathrm{s}$ in the cases investigated. A force balance model has sufficient accuracy to describe the acceleration and detachment in the particle-cavity system. This phenomenon has a bolder perspective: With strong acoustic transients, able to accelerate particles by this generic mechanism of self-propulsion, the technique if downscaled to submicron sized particles might allow for a novel method of drug delivery into biological cells. Particles coated with or consisting of a specific drug could be sonically propelled into neighboring cells or tissue.

Experiment.-The experimental setup, depicted in Fig. 1, consists of the shock wave source, a polystyrene flask (Nalge Nunc $50 \mathrm{ml}$ ) containing the suspension of particles, the imaging and illumination devices, and digital delay lines. A single finite amplitude wave is generated by a focused piezoelectric source; it is a slightly modified commercial extracorporeal lithotripter Piezolith 3000
(Richard Wolf GmbH, Knittlingen, Germany). The diameter of the shock wave source is $255 \mathrm{~mm}$ and the focusing angle $83^{\circ}$. The axis of the source is placed at an angle of $45^{\circ}$ to the horizontal plane at the bottom of a container. The container and the flask are filled with filtered, deionized, and degassed water $\left(\mathrm{O}_{2}\right.$ concentration $3.3 \mathrm{mg} / 1$ of water) at room temperature. The pictures are taken with a sensitive slow scan CCD (charged coupled device) camera (Imager $3 \mathrm{~S}$ with $320 \times 256$ pixels at a binning mode of $2 \times 2$ and $9 \mu \mathrm{m}$ pixel size, LaVision $\mathrm{GmbH}$, Germany) equipped with a long distance microscope (K2, CF4 objective, Infinity, USA). The microscope operates from a working distance of $45 \mathrm{~mm}$ giving a maximum resolution of $3.4 \mu \mathrm{m}$ per binned pixel. The CCD camera is operated in a double-frame mode, which allows two images to be taken in rapid succession before they are transferred to a computer. Both frames are strobe illuminated with a light emitting diode for exposure times of $1.8 \mu \mathrm{s}$.

Figure 2 displays a typical pressure recording, taken with a fiber optical hydrophone FOP-500 (Dr. Pecha, University of Stuttgart, see Ref. [11]) for a discharge voltage set to $5 \mathrm{kV}$. The tensile stress wave, which at

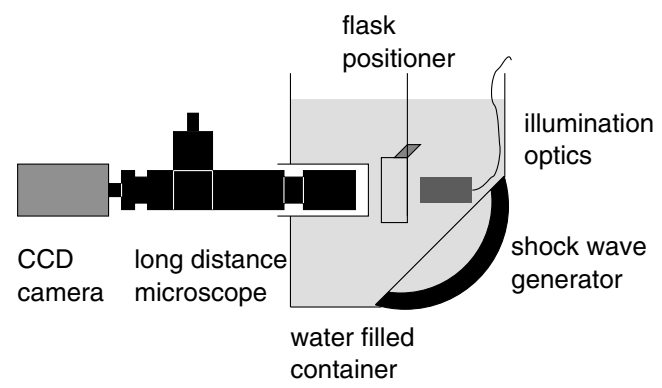

FIG. 1. Sketch of the experimental setup: The microscope, embedded partly in a cylindrical glass housing, is operated at a working distance of $45 \mathrm{~mm}$ from the focus of the shock wave generator. The flask is positioned with an $x y z$-translation stage. 


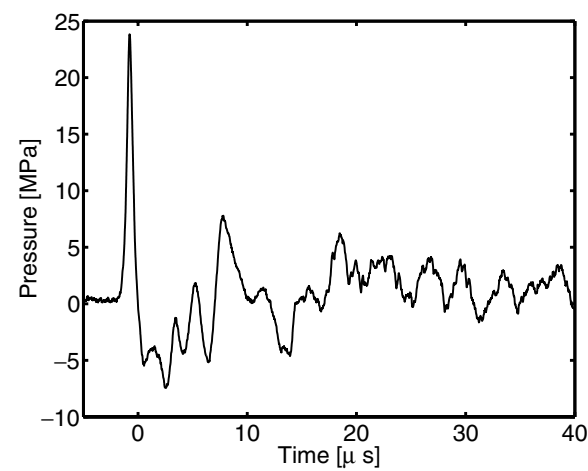

FIG. 2. Pressure, $P(t)$, versus time recorded with a fiber optical hydrophone.

time $t=0$ follows the finite amplitude wave front, causes cavitation nuclei in the flask (and also within the main container) to expand, and at locations where they grow beyond critical size they explode into vaporous cavities.

The flask contains filtered and degassed water seeded with globally spherical, hydrophilic particles. Two different batches of such particles have been subjected to the tensile stress wave. None of the batches caused cavitation events at discharge voltages of $3 \mathrm{kV}$, corresponding to a peak tensile stress of $3 \mathrm{MPa}$, but at $4 \mathrm{kV}$ some events were observed for the batch of polystyrene particles in Fig. 3(a), and at $5 \mathrm{kV}$, corresponding to a peak tensile stress of $-7 \mathrm{MPa}$, chosen for the main experiments, these particles often caused cavitation. Even at this high stress level no cavitation events occurred with the acrylic polymer particles shown in Fig. 3(b). The essential difference was their surface structure: particles causing cavitation had a corrugated surface [Fig. 3(a)], while particles with a smooth surface [Fig. 3(b), for details see [9]] did not cause cavitation.

The further experiments were performed using the polystyrene particles [Fig. 3(a)]. Many of these caused cavitation, but certainly not all of them. This is understood from the observation, that some of them had a highly corrugated surface [Fig. 3(a)], others had a relatively smooth surface. In each experiment the camera was first operated in a continuous mode, displaying the mo-
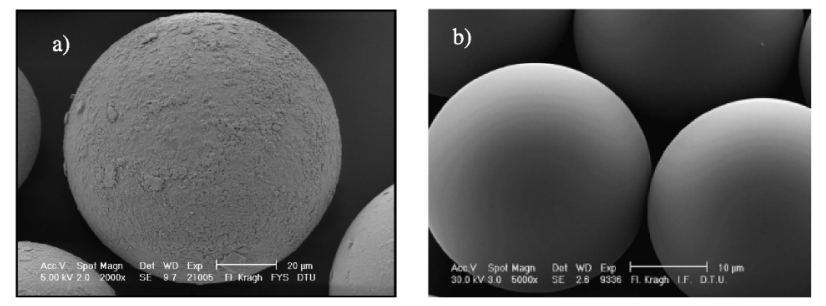

FIG. 3. Scanning electron microscope (SEM) pictures of the particles taken from batches where (a) cavitation inception was observed (copolymer:divinylbenzol, diameter distribution 30 to $150 \mu \mathrm{m}$ ), and (b) no inception was achievable (monodisperse $30 \mu \mathrm{m}$ dynospheres EXP-SS-42.3-RSH, see Ref. [9]). Please note the different magnifications. tion of the particles (density $\rho_{p}=1.07 \times 10^{3} \mathrm{~kg} / \mathrm{m}^{3}$ ) due to gravity and secondary flow. When a particle moved into the focal zone, the first frame was taken, automatically the camera switched to the double framing mode, and the shock wave generator was activated. The first frame was taken about half a second before the doubleframe sequence, which had an interframe time adjustable from 0.4 to several $\mu \mathrm{s}$.

Two typical sequences of explosively expanding cavities on particles are depicted in Figs. 4(a) and 4(b). Figure 4(a) displays three particles indicated with arrows, which are slowly sinking in the top frame. The second frame is taken just after the arrival of the tensile wave. Expanding cavities attached to two of the particles are visible, captured in motion, and blurred due to the finite exposure time. $6.2 \mu \mathrm{s}$ later these cavities have expanded further and the particles are accelerated in directions opposite to the cavity growth. The shock wave arrives from below, and at an angle of $45^{\circ}$ from behind the picture plane. The directions of the particle ejection are seen to be stochastic and independent of the shock wave direction.

The process of detachment of the particle from the cavity is visualized in Fig. 4(b) where the fast frames are taken at later times: Again the undisturbed particle is depicted in the top frame. In the next one, taken $8 \mu$ s after the tensile stress wave has arrived, an attached cavity of radius $150 \mu \mathrm{m}$ has developed. Here, the particle has moved away from the bubble, thereby forming a necklike structure connecting the cavity with the particle. In the last frame of Fig. 4(b), taken $24 \mu$ s after the stress wave

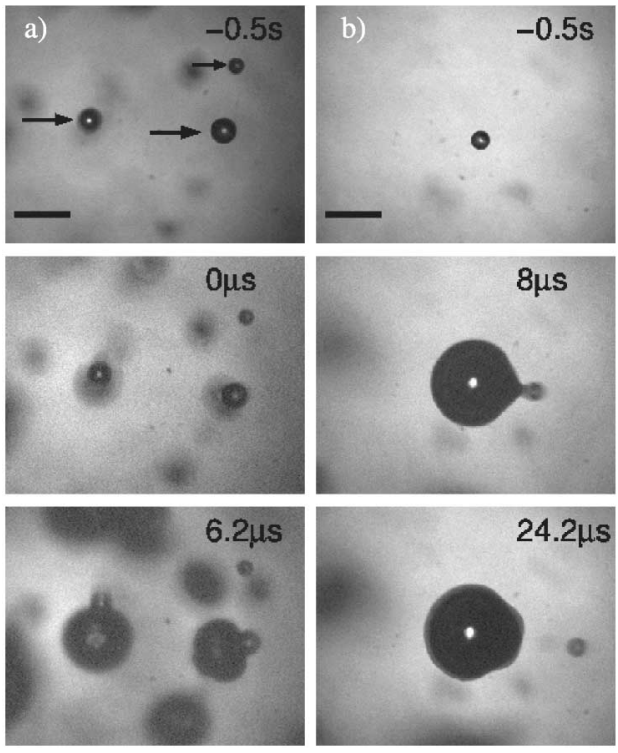

FIG. 4. Two three-frame sequences (a) and (b) depicting the explosive growth of cavitation bubbles from particles and their later separation. The timing of the individual frames relative to the start of the tensile wave (see Fig. 2) are indicated at the top of each frame (the length of the bar is $200 \mu \mathrm{m}$ ). 
arrival, the cavity has expanded to a radius of $170 \mu \mathrm{m}$ and the neck between the particle and the cavity eventually has broken. Thereby, a surface wave has been excited and propagates on the cavity surface.

Force balance model. - Let us consider a cavity that develops in water from a cavitation nucleus on the surface of an almost spherical solid particle of radius $R_{p}$ when it is exposed to the stress wave from the lithotripter. Such a wave is shown in Fig. 2. The pressure pulse $P(t)$ is superposed on the atmospheric pressure $P_{0}$ to give the far field pressure $P_{\infty}(t)=P_{0}+P(t)$. The tensile part of the stress perturbation is initiated at the time $t=0$. We approximate $P(t)$ with the measured data.

For simplicity we assume that when the tensile strength of the liquid-particle system is exceeded at the time $t=$ $t_{\text {crit }}$ an attached spherical cavity of radius $\left(R_{c}\right)_{\text {crit }}$ is developed, and that $\left(d R_{c} / d t\right)_{\text {crit }} \approx 0$. We assume that the dynamics of the cavity is governed by the Rayleigh-Plesset equation

$$
R_{c} \frac{d^{2} R_{c}}{d t^{2}}+\frac{3}{2}\left(\frac{d R_{c}}{d t}\right)^{2}=\rho_{l}^{-1}\left[P_{v}-\frac{2 \sigma}{R_{c}}-P_{\infty}(t)\right],
$$

where $\rho_{l}=10^{3} \mathrm{~kg} / \mathrm{m}^{3}$ is the density of the liquid, $\sigma=$ $7.3 \times 10^{-2} \mathrm{~kg} / \mathrm{s}^{2}$ is the surface tension, and $P_{v}=3.2 \mathrm{kPa}$ is the vapor pressure.

During the growth of a cavitation nucleus, the pressure inside the void very quickly drops to the vapor pressure $P_{v}$. When it reaches critical size it grows explosively and becomes an attached spherical cavity as assumed above. A stress relaxation wave is released, and the pressure drops in radial direction from $P_{v}$ at the cavity surface to $P_{\infty}$ in the far field. At first the particle's far side is shielded from the stress relaxation wave, and the particle is accelerated radially with the cavity surface. As long as the far field tensile stress increases, the particle moves with the velocity of the cavity surface at the contact point. However, at the time $t_{\text {sep }}$ when the rate of expansion of the cavity passes its maximum, the particle detaches from the cavity and moves on through the liquid, at first with the maximum speed acquired, but the detachment itself and frictional forces decelerate the particle motion.

In the time interval $t_{\text {crit }}<t<t_{\text {sep }}$ no external forces influence the cavity-particle system, and $\sum[d(m u) / d t]=$ 0 is required. The symbol $m$ stands for the mass of the particle as well as for the added masses of the particle and the cavity. With the particle initially at rest, the growth of a single cavity from a small area of the particle surface implicates that the expanding cavity and the particle move in opposite directions at increasing velocities, $u_{c}$ and $u_{p}$, respectively. The cavity is assumed to expand spherically. Thus, the particle acquires an increasing translational momentum. The motion of the center of the expanding cavity in the opposite direction results in a momentum of the same numerical size, related to its added mass. When the tensile stress in the lithotripter pulse has passed its maximum, the radial velocity $v_{R}$ of the cavity surface begins to decrease, but the particle velocity remains almost unaffected, i.e., the particle starts moving away from the cavity.

From cavitation inception at critical tensile stress when $t=t_{\text {crit }}$ until the moment of separation of the particle from the cavity at $t=t_{\text {sep }}$ momentum balance demands

$$
\frac{d}{d t}\left[u_{c}\left(\frac{1}{2} \rho_{l} \frac{4}{3} \pi R_{c}^{3}\right)+u_{p}\left(\rho_{p} \frac{4}{3} \pi R_{p}^{3}\right)\right]=0 .
$$

In Eq. (2) the particle has no added mass, as it is assumed that it moves with the velocity of the liquid at the cavityparticle contact point. However, at times $t>t_{\text {sep }}$ an added mass $d / d t\left(1 / 2 \rho_{l} \Delta u \frac{4}{3} \pi R_{p}^{3}\right)$ has to be accounted for. Here $\Delta u=u_{p}-u_{l}$, in which the velocity of the liquid $u_{l}$ is evaluated at the center of the particle. The particle-cavity contact condition gives

$$
u_{c}+\frac{d R_{c}}{d t}=u_{p} .
$$

Integrating Eq. (2) we obtain

$$
u_{p}=\left(\frac{d R_{c}}{d t}\right)\left(1+\frac{2 R_{p}^{3} \rho_{p}}{R_{c}^{3} \rho_{l}}\right)^{-1},
$$

and

$$
u_{c}=-\left(\frac{d R_{c}}{d t}\right)\left(\frac{R_{c}^{3} \rho_{l}}{2 R_{p}^{3} \rho_{p}}+1\right)^{-1} .
$$

When the radial expansion of the cavity decelerates, the particle is no longer pushed by the cavity wall, but detaches with the momentum gained. Thus, $t=t_{\text {sep }}$ is the time when $\left(d^{2} R_{c} / d t^{2}\right)$ changes sign from positive to negative and $\Delta u$ becomes positive instead of being zero. From this time the analysis stated in Eqs. (3)-(5) is not valid anymore: the forces due to drag and added mass acting on the particle need to be incorporated. The drag force, $F_{D}$ is calculated from the relative velocity $\Delta u$ of the particle, $F_{D}=\frac{1}{2} C_{D} \rho_{l} \Delta u^{2} \pi R_{p}^{2}$, with the drag coefficient, $C_{D}$, depending on the Reynolds number. However, it should be noted that it is not a steady flow, but one that builds up from potential flow into turbulent separation, see [12].

Figure 5 presents the calculated motions of the cavity and the particle of Fig. 4(b).The positions of the centers of the cavity and the particle are shown as left and right dotted lines, respectively, as calculated for the measured pressure pulse (Fig. 2). The initial bubble radius is taken as $R(t=0)=50 \mathrm{~nm}$. Explosive growth sets in at $t_{\text {crit }}=$ $0.34 \mu \mathrm{s}\left(P_{\infty}=-2.8 \mathrm{MPa}\right)$. The diameter of the particle and the cavity are represented by the width of the dark and light shaded regions, respectively. Until the time of separation at $t_{\text {sep }}=5.0 \mu$ s the particle and cavity stay in contact. Subsequently the particle detaches at a speed of $38 \mathrm{~m} / \mathrm{s}$. During the initial growth of the cavity its center moves approximately $62 \mu \mathrm{m}$ to the left due to the conservation of momentum, and the cavity collapses at 


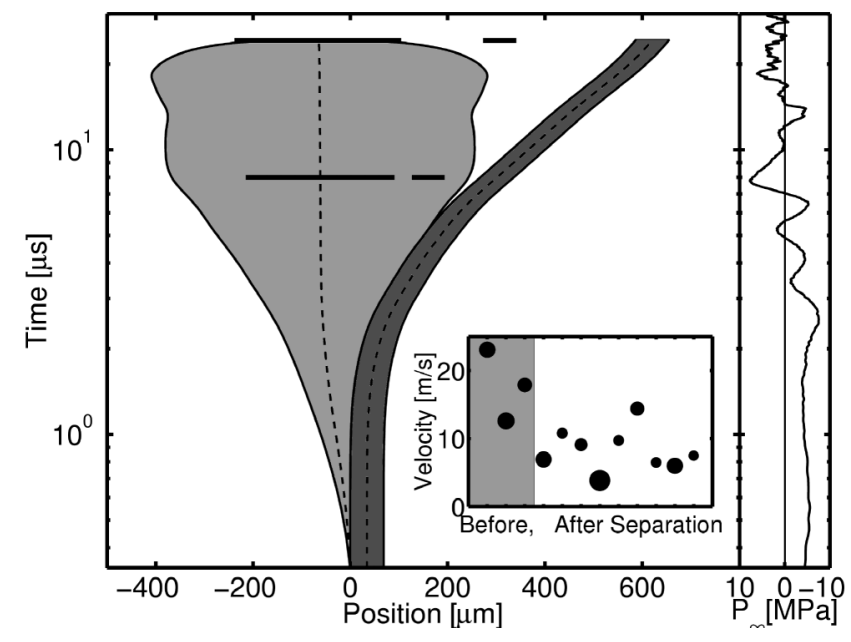

FIG. 5. Position and size of the particle-cavity system versus time (note the logarithmic scale). Left and right dotted lines show calculated positions of the cavity and particle centers, respectively; left and right shaded regions represent the diameters of the cavity and the particle, respectively. The bars indicate the position and the size of the cavity (left) and the particle (right) from the experiment in Fig. 4(b) (lower two frames). The measured pressure profile is shown along the right edge of the graph. The inset depicts the measured velocity of the particles for multiple experimental runs before and after separation from the cavity. The size of the disk symbols scales linearly with the particle diameter from 56 to $108 \mu \mathrm{m}$.

$t \approx 24 \mu \mathrm{s}$. The experimentally recorded positions and sizes of the cavity and particle diameter from Fig. 4(b) are plotted as solid bars. Qualitative agreement is seen, but quantitatively only the order of magnitude is correct. This is not unexpected as we assume a too simple model for the inception and bubble dynamics. The right-hand side of (1) is overestimated already when the cavity becomes of size as the particle. Further, in the experiments the bubble growth is affected by the presence of nearby expanding bubbles. Thus, the velocity field surrounding the bubble becomes important and may explain the smaller bubble radius, and thereby the lower translatory velocity of the particle measured after separation. The inset of Fig. 5 depicts the measured velocities obtained from multiple runs where the particle motion could be captured sharply in the focal plane. The highest velocities are measured before the particle separates from the cavity.

Discussion and conclusions.-The finding that a selfpropelled particle accelerator results from cavity nucleation can be considered a generic process: Whenever a cavity grows rapidly from the surface of a small particle, the particle eventually detaches at high speed from the cavity which it has itself nucleated.

This is a result that is important in connection with the study of cavitation nuclei. The discussion of their nature-stabilized spherical gas bubbles or surface nuclei on particles - has been ongoing. Though $[3,5,8,9]$ point to the latter ones, the direct observation of cavity-particle separation has been missing until now. Further, the particle ejection suggests that accelerated particles may penetrate into nearby soft surfaces, e.g., biological tissue or cells during exposure to strong, focused sound fields. A possible beneficial application might be the acceleration of micro- or nanometer-sized particles, made from or coated with specific drugs, at exposure to strong transient, ultrasound waves in a suspension of biological cells. The accelerated particles may permeate cell membranes and deposit the drugs (e.g., toxins) into the cytoplasm of the cells. However, for smaller sized particles the energy needed to form the gaseous neck [see second frame in Fig. 4(b)] could be higher than the kinetic energy of the particle, thus preventing the particle from separating from the cavity. To estimate the energy loss, we assume that during separation a cylindrical neck with a radius $r_{n}$ and length $x$ is created. Equating the kinetic energy of the particle with the surface energy of the neck, $\int_{0}^{x} 2 \pi \sigma r_{n} d x=2 / 3 \pi \rho_{p} u_{p}^{2} r_{p}^{3}$ allows us to estimate the maximum achievable length of the neck as $x=$ $1 / 3 \pi \sigma \rho_{p} u_{p}^{2} r_{p}^{3} / r_{n}$. Additionally separation of the particle from the cavity depends on the stability of the neck.

Possible damage effects of the cavitation mechanism considered here are governed by the growth phase of the bubbles in contrast to the well-established damage due to the collapse of cavitation bubbles where microjetting, high pressure, and temperature are responsible.

The authors gratefully acknowledge inspiring discussions with Detlef Lohse (U Twente) and the preparation of SEM pictures by Flemming Kragh, the Center of Quantum Protein, TU Denmark. The work was supported by FOM (The Netherlands) under Grants No. 99MS07 and No. 02PMT04.

*Electronic address: c.d.oh1@tnw.utwente.nl

[1] V. P. Skripov, Metastable Liquids (John Wiley and Sons, New York, 1974).

[2] F. E. Fox and K. F. Herzfeld, J. Acoust. Soc. Am. 26, 984 (1954).

[3] E. N. Harvey, D. K. Barnes, W. D. McElroy, A. H. Whiteley, D. C. Pease, and K.W. Cooper, J. Cell Comp. Physiol. 24, 1 (1944).

[4] R. E. Apfel, Ultrasonics 22, 167 (1984).

[5] M. Greenspan and C. E. Tschiegg, J. Res. Natl. Bur. Stand. Sect. C 71C, 299 (1967).

[6] M. Strassberg, J. Acoust. Soc. Am. 31, 163 (1959).

[7] R. E. Apfel, J. Acoust. Soc. Am. 48, 1179 (1970).

[8] K. A. Mørch, J. Fluids Eng. 122, 494 (2000).

[9] H. Marschall, K. A. Mørch, A. P. Keller, and M. Kjeldsen, Phys. Fluids 15, 545 (2003).

[10] M. Holmberg, A. Kühle, J. Garnæs, K. A. Mørch, and A. Boisen, Langmuir 19, 10510 (2003).

[11] J. Staudenraus and W. Eisenmenger, Ultrasonics 31, 267 (1993).

[12] L. Prandtl, J. R. Aeronaut. Soc. 31, 730 (1927). 